archives

of thermodynamics

Vol. 33(2012), No. 3, 133-145

DOI: $10.2478 / v 10173-012-0024-x$

\title{
A new method for optimum heating of pressure components in supercritical steam power blocks
}

\section{DARIUSZ RZĄSA \\ PIOTR DUDA*}

Cracow University of Technology, Faculty of Mechanical Engineering, al. Jana Pawła II 37, 31-864 Kraków, Poland

\begin{abstract}
One of the major concerns of the power energy industries is a proper operation of steam power blocks. Pressurized working medium and high temperature cause very high stresses in the construction elements such as collectors, separators or steam valves. They are exposed to sudden temperature and pressure changes that cause high stresses at certain points. Additionally, the cyclic character of loading causes material fatigue, known as low-cyclic fatigue, which may lead to the formation of fracture. Thus, methodologies offered by many companies should ensure reliable and safe operation of steam power blocks. The advanced numerical solutions for determining time-optimum medium temperature changes are presented. They are based on Levenberg-Marquardt and nonlinear programming by quadratic Lagrangian methods. The methods allow us to find parameters for start-up and shut-down operation that can reduce total stresses to limits governed by European regulations. Furthermore, the heating and cooling operations are conducted in a shortest time possible.
\end{abstract}

Keywords: Optimization methods; Total stresses; Steam boilers; Heat transfer

\section{Nomenclature}

$\begin{array}{lll}\mathbf{H}, \mathbf{J}, \delta & - & \text { matrices } \\ \mathbf{x} & - & \text { vector of searched parameters } \\ k & - & \text { thermal conductivity, } \mathrm{W} /(\mathrm{m} \mathrm{K})\end{array}$

\footnotetext{
${ }^{*}$ Corresponding Author. E-mail address: pduda@mech.pk.edu.pl
} 


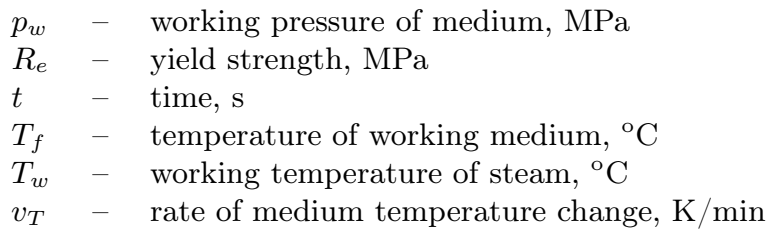

\section{Greek symbols}

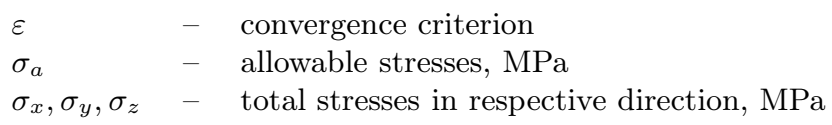

\section{Introduction}

The transient operation of the power block devices cause high stresses in the construction elements. These stresses originate from very high working pressure and temperature gradient change during operation. Therefore, the operation should be carried out in such a manner that the total stresses do not exceed the allowable limit.

It is important to find the optimum working medium temperature history during transient operations. The optimum temperature history should assure that the maximum total stresses are within the allowable stress limit and the transient operation is conducted in the shortest time possible. The regulations such as German boiler code - Technische Regeln für Dampfkessel 301 (TRD) [1] allow us to find the working medium temperature history. However, the TRD 301 procedure is based on the quasi-steady, one dimensional temperature distribution in the whole component. Since the heating and cooling processes are transient operations the quasi-steady state often does not occur. Furthermore, the complicated geometry of the component causes difficulties in assessing the regions with the highest stresses. Thus, the operations conducted according to the TRD 301 code, may account for the state, where the allowable stresses are exceeded.

Paper [2] shows the numerical method based on the golden search method, which could be used to find the optimum medium temperature so that the maximum absolute thermal stresses in the whole construction element would not exceed the allowable stresses. Paper [3] presents the method for determining the optimum medium temperature, which ensures that the sum of the thermal stresses and stresses caused by pressure at selected points do not exceed the allowable stresses. The presented optimum medium temperature consists of the initial medium temperature step and later increases with the 
optimum rate of temperature change. The extended version of paper [3] was published in 2010 [4]. Papers [5,6] present the numerical optimization procedure, based on the Levenberg-Marquardt algorithm that allows finding the optimum medium temperature history. The aim of this paper is to present and compare two different optimization methods, the first one based on the Levenberg-Marquardt approach (L-M) and the second one based on nonlinear programming by quadratic Lagrangian (NLPQL) algorithms [7-9] that allow us to find the optimum temperature histories, so that the total stresses during the start-up processes are kept at an acceptable level.

\section{Mathematical formulation of optimization procedures}

Consider a heating process, where fluid enters the inner space of a construction element that has a uniform temperature $T_{0}$. Fluid has an initial temperature $T_{f_{1}}$ and then fluid temperature rises with a constant rate of temperature change $v_{T_{1}}$ until the nominal working medium pressure is reached. Next, the temperature of the medium changes stepwise to a value $T_{f_{2}}$ and later rises with a constant rate of change of temperature $v_{T_{2}}$. Thus the working medium temperature history is defined by four parameters.

Due to the high internal pressure and temperature gradients across the structural element, high stress concentration areas occur on the inner surface of the construction element [11,12]. The objective is to choose the optimum parameters, i.e. the optimum rates of fluid temperature change $v_{T_{1}}, v_{T_{2}}$ and the optimum fluid temperature steps $T_{f_{1}}, T_{f_{2}}$, in such a way that the heating process is conducted in the shortest time and the allowable stresses $\sigma_{a}$ are kept at an acceptable level.

\subsection{Levenberg-Marquardt method}

The Levenberg-Marquardt (L-M) optimization algorithm [13] was employed in order to find the time-optimum medium temperature history. The optimum parameters $T_{f_{1}}, v_{T_{1}}$ and $T_{f_{2}}, v_{T_{2}}$ are found when the following equation is satisfied:

$$
\sigma_{a}-\sigma_{\max }\left(T_{f_{1}}, v_{T_{1}}, \mathrm{~T}_{f_{2}}, v_{T_{2}}, t_{i}\right) \cong 0, \quad i=1, \ldots, m
$$

where $\sigma_{\max }$ denotes the highest value of the compressive stresses component and $m$ is the number of time steps during the heating process. In other 
words, minimizing the sum

$$
S(x)=\sum_{i=1}^{m}\left[\sigma_{a}-\sigma_{\max }\left(T_{f_{1}}, v_{T_{1}}, T_{f_{2}}, v_{T_{2}}, t_{i}\right)\right]^{2} \cong 0, \quad i=1, \ldots, m
$$

allows the optimum parameters to be established.

The updating of the searched parameters at every $k$-th iteration step is performed based on the following rule

$$
\mathbf{x}^{(k+1)}=\mathbf{x}^{(k)}+\delta^{(k)},
$$

The unknown parameters of $\mathbf{x}$ are: $x_{1}=T_{f_{1}}, x_{2}=v_{T_{1}}, x_{3}=T_{f_{2}}, x_{4}=v_{T_{2}}$, where

$$
\delta^{(k)}=\left(\mathbf{H}^{(k)}+\lambda \operatorname{diag} \mathbf{H}^{(k)}\right)^{-1}\left(\mathbf{J}^{(k)}\right)^{T}\left[\sigma_{a}-\sigma_{\max }\left(x^{(k)}\right)\right], k=0,1, \ldots,
$$

The $\mathbf{H}$ and $\mathbf{J}$ are called the Hessian and Jacobian matrices, respectively. They can be expressed in the following way

$$
\mathbf{J}^{(k)}=\left.\frac{\partial \sigma_{\max }(x)}{\partial x^{T}}\right|_{x=x^{(k)}}=\left.\left[\begin{array}{lll}
\frac{\partial \sigma_{1}}{\partial x_{1}} & \ldots & \frac{\partial \sigma_{1}}{\partial x_{n}} \\
\cdots & \cdots & \cdots \\
\ldots & \ldots & \ldots \\
\frac{\partial \sigma_{m}}{\partial x_{1}} & \cdots & \frac{\partial \sigma_{m}}{\partial x_{n}}
\end{array}\right]\right|_{x=x^{(k)}}
$$

and

$$
\mathbf{H}^{(k)}=\left.\frac{\partial^{2} \sigma_{\max }(x)}{\left(\partial x^{T}\right)^{2}}\right|_{x=x^{(k)}}=\left.\left[\begin{array}{lll}
\frac{\partial^{2} \sigma_{1}}{\partial x_{1} \partial x_{1}} & \cdots & \frac{\partial^{2} \sigma_{1}}{\partial x_{1} \partial x_{n}} \\
\cdots & \cdots & \cdots \\
\cdots & \cdots & \cdots \\
\frac{\partial^{2} \sigma_{m}}{\partial x_{n} \partial x_{1}} & \cdots & \frac{\partial^{2} \sigma_{m}}{\partial x_{n} \partial x_{n}}
\end{array}\right]\right|_{x=x^{(k)}}
$$

The solution for the optimum parameters is obtained if the assumed convergence criterion

$$
x_{i}^{(k+1)}-x_{i}^{(k)} \leq \varepsilon, \quad i=1, \ldots, n
$$

is fulfilled. 


\subsection{Nonlinear programming by quadratic Lagrangian method}

The presented optimization algorithm for finding time-optimum temperature history uses a successive quadratic programming method to solve the general nonlinear programming problem [7]. A nonlinear optimization problem with inequality constraints is considered. The goal is to find a design vector $\mathbf{x}$ which minimizes the objective function defined as a total time of start-up operation

$$
S(\mathbf{x})=t_{t o t}
$$

subject to

$$
\begin{gathered}
g(\mathbf{x})=\sigma_{\max _{i}}(\mathbf{x})-\sigma_{a} \geq 0, \quad i=1,2, \ldots, t_{t o t} \\
\mathbf{x}_{l} \leq \mathbf{x} \leq \mathbf{x}_{u}
\end{gathered}
$$

where $t_{\text {tot }}$ denotes the total time of start-up operation, $\sigma_{\max _{i}}$ is the highest value of the compressive stresses component at time step $i$ during the heating process, and $\sigma_{a}$ stands for maximum allowable stress value. The unknown parameters of $\mathbf{x}$ are denoted as $x_{1}=T_{f_{1}}, x_{2}=v_{T_{1}}, x_{3}=T_{f_{2}}$, $x_{4}=v_{T_{2}}$. The updating of the unknown parameters at every $k$ th iteration is performed based on the following rule:

$$
\mathbf{x}^{(k+1)}=\mathbf{x}^{(k)}+\lambda \mathbf{s}^{(k)},
$$

where $\mathbf{x}^{(k)}$ denotes the current iteration, and $\lambda$ is the optimal step length along the direction $\mathbf{s}$. The method obtains subproblems by using a quadratic approximation of the Lagrangian and by linearizing the constraints. Thus, the subproblem can be formulated as follows: find $\mathbf{s}$ which minimizes

$$
q(\mathbf{s})=\nabla f(\mathbf{x})^{T} \mathbf{s}+\frac{1}{2} \mathbf{s}^{T} \mathbf{H} \mathbf{s}
$$

subject to

$$
\begin{gathered}
g_{j}(\mathbf{x})+\nabla g_{j}(\mathbf{x})^{T} \mathbf{s} \geq 0, \quad j=1,2, \ldots, m \\
\mathbf{x}_{l}-\mathbf{x}_{k} \leq \mathbf{s} \leq \mathbf{x}_{u}-\mathbf{x}_{k},
\end{gathered}
$$

where $\mathbf{H}$ is a positive definite approximation of the Hessian. 

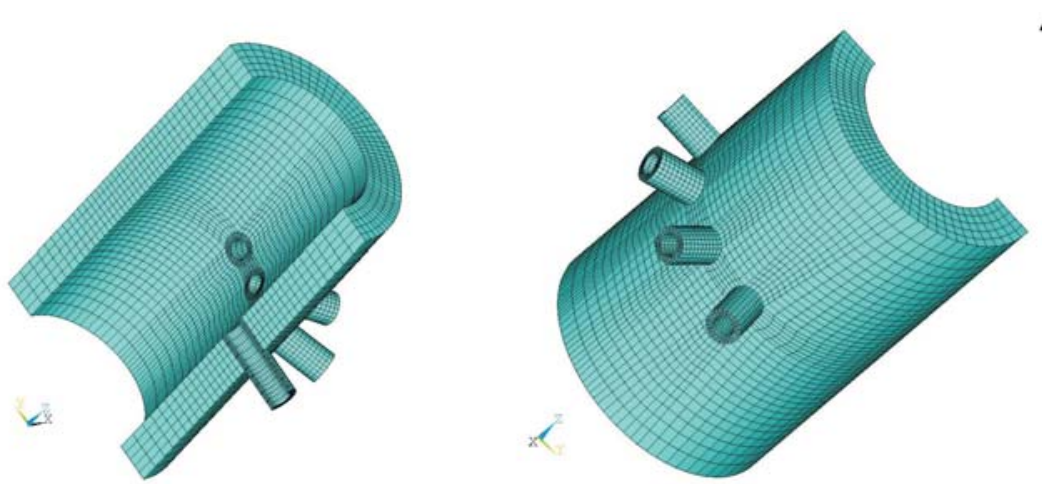

Figure 1. The outlet header divided into finite elements.

\section{Numerical simulation of heating operations}

The numerical analysis of start-up operation is presented for an outlet header. This component is mounted in power units of $460 \mathrm{MW}$. The geometry is presented in Fig. 1. The outlet header is designed for pressure $p_{w}=29 \mathrm{MPa}$ and steam temperature $T_{w}=559{ }^{\circ} \mathrm{C}$. The extreme working conditions of the outlet header require special alloy steel to be employed in the construction. One of the widely used construction materials for the power plant elements is the ferritic alloy steel, X10CrMoVNb9-1 (P91) [10]. The following material properties were assumed for the calculations: Young modulus $E=196112 \mathrm{MPa}$, thermal expansion coefficient $\beta=1.12^{-5} 1 / \mathrm{K}$, specific heat $C_{p}=640.1 \mathrm{~J} /(\mathrm{kgK})$, thermal conductivity $\lambda=29.7 \mathrm{~W} /(\mathrm{mK})$, density $\rho=7750 \mathrm{~kg} / \mathrm{m}^{3}$, Poisson's ratio $v=0.3$. The heat transfer coefficient $\alpha=2000 \mathrm{~W} /\left(\mathrm{m}^{2} \mathrm{~K}\right)$ on the heated surface in subsequent analysis was constant over the entire process time. The outer surface was perfectly isolated, so there was no heat exchange between the heated component and the surrounding environment.

\subsection{Heating based on TRD regulations}

The German boiler regulations - Technische Regeln für Dampfkessel 301 (TRD) guide on how to operate steam boilers. These regulations define temperature and pressure histories of working medium during start-up and shut down operation. The temperature history is described by two parameters $v_{T_{1}}$ and $v_{T_{2}}$. The parameters $v_{T_{1}}$ and $v_{T_{2}}$ denote rates of temperature change at the beginning and at the end of the process, respectively. For 
heating operation of outlet header the following parameters were found: $v_{T_{1}}=7.09 \mathrm{~K} / \min v_{T_{2}}=18.85 \mathrm{~K} / \mathrm{min}$. The pressure of working medium changes as a function of temperature. Figure 2 illustrates the complete pressure and temperature history based on the German boiler regulations. The maximum compressible stresses during start-up operation from the cold state should not exceed the allowable stress $\sigma_{a}=-126.7 \mathrm{MPa}$.
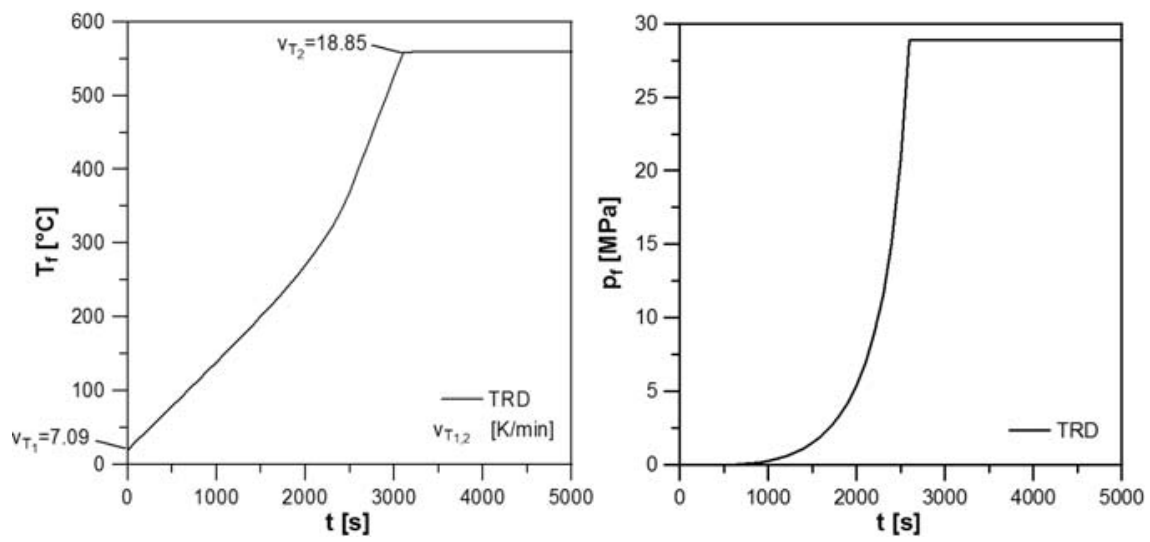

Figure 2. Temperature (left) and pressure (right) history during the heating operation based on TRD regulation.

The start-up operation of outlet header was simulated with the aid of Ansys program, which is based on finite element method (FEM). The outlet header geometry was divided into 8-node linear bricks element as illustrated in Fig. 1. During the start-up process the working medium at a temperature of $20^{\circ} \mathrm{C}$ flows into the outlet header that has a uniform temperature $T_{0}=$ $20^{\circ} \mathrm{C}$. Next, the medium temperature changes with the calculated rates of temperature change $v_{T_{1}}$ and $v_{T_{2}}$ until working parameters $p_{w}=29 \mathrm{MPa}$ and $T_{w}=559^{\circ} \mathrm{C}$ were reached. It can be seen from Fig. 2 that the total time of heating operation took about 3200 s. During the heating process the total stresses in the whole construction element were monitored. Figure 3 presents the maximum total compressive stress history. The highest stress value reads $-233 \mathrm{MPa}$ at the time $t=3100 \mathrm{~s}$ and exceeds the stress limit, $\sigma_{a}$, by more than $80 \%$. Maximum compressive stresses are located on the inner surface of outlet header at the edge as presented in Fig. 4. 


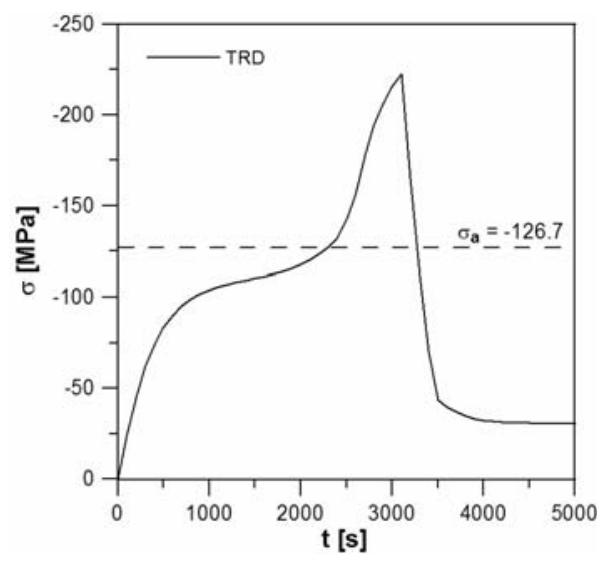

Figure 3. Maximum total compressive stress history during the start-up process according to TRD 301 regulations.
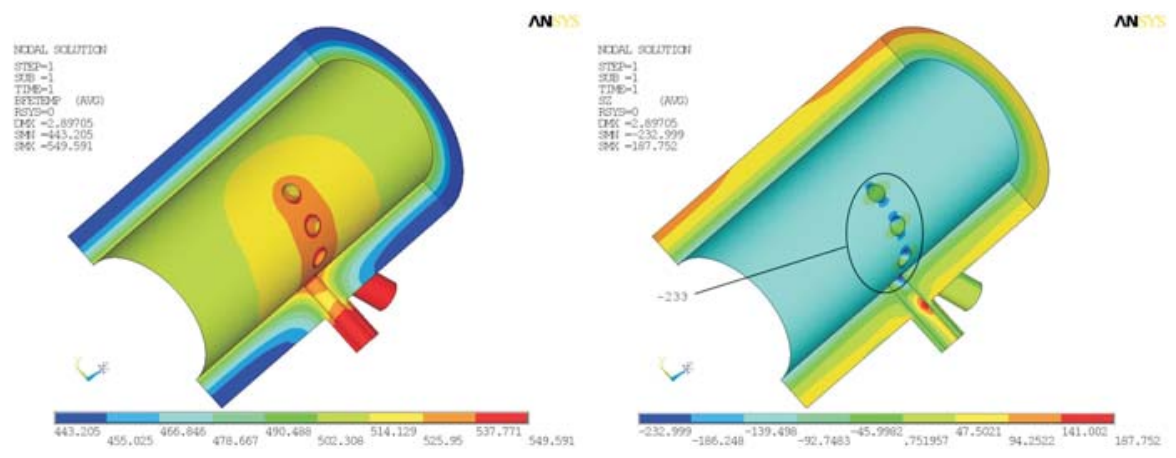

Figure 4. Temperature distribution in ${ }^{\circ} \mathrm{C}$ (left) and maximum compressive stress $\sigma_{z}$ distribution in $\mathrm{MPa}$ (right) on the inner surface at time $t=3100 \mathrm{~s}$.

\subsection{Heating based on Levenberg-Marquardt algorithm}

In this stage the optimization Levenberg-Marquardt algorithm was used in order to find the optimum temperature history for start-up operation. The temperature history is defined by four parameters calculated by L-M algorithm: $T_{f_{1}}=64.6{ }^{\circ} \mathrm{C}, v_{T_{1}}=7.9 \mathrm{~K} / \mathrm{min}, T_{f_{2}}=442.5{ }^{\circ} \mathrm{C}, v_{T_{1}}=10.9 \mathrm{~K} / \mathrm{min}$. It was assumed that the pressure of working medium changes along the saturation line. The complete temperature and pressure history is shown in Fig. 5. 

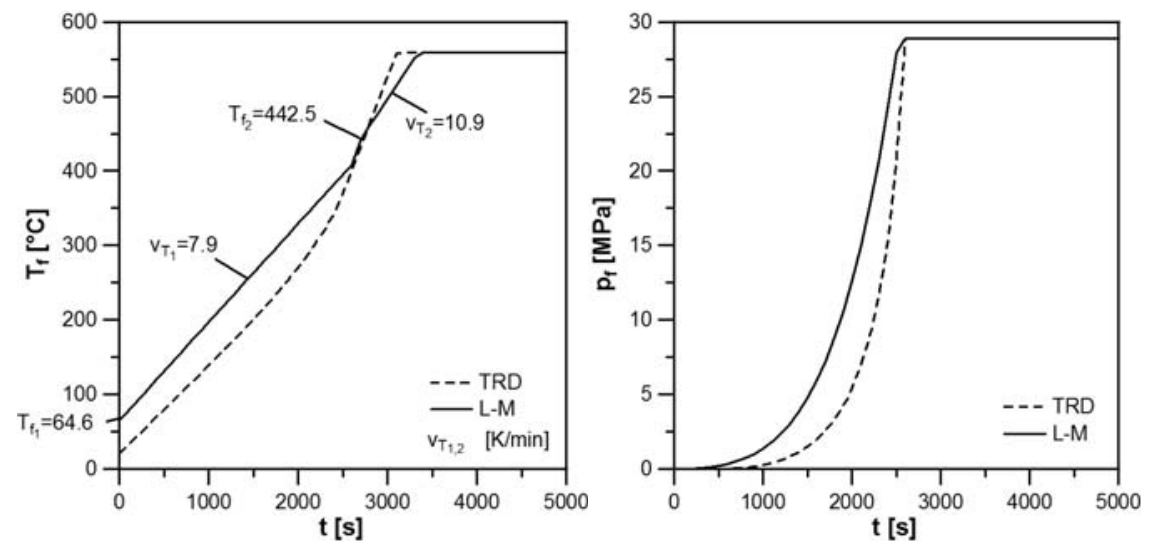

Figure 5. Comparison of temperature (left) and pressure (right) histories during the heating operation based on L-M algorithm and TRD 301 regulations.

For the temperature and pressure histories presented in Fig. 5 , numerical simulation of the heating process of outlet header was carried out. The outlet header discrete geometry was used as in the previous case and is presented in Fig. 1. At the cold state, i.e. at the beginning of the heating operation, outlet header had a uniform temperature $T_{0}=20^{\circ} \mathrm{C}$. Next, the working medium at a temperature $T_{f_{1}}$ entered the outlet header and began to rise with a constant rate of temperature change $v_{T_{1}}$. After the operation time $t=2800 \mathrm{~s}$ the working medium temperature was changed stepwise to the temperature $T_{f_{2}}$, and temperature change rate was increased to value $v_{T_{2}}$. The working parameters were reached after the time $t=3350 \mathrm{~s}$. During heating operation the total compressive stresses were monitored. The maximum total compressive stress history together with the stress history from previous case is plotted in Fig. 6. The maximum total compressive stresses $\sigma_{\max }=-128.5 \mathrm{MPa}$ occurred on the inner surface at time $t=2800 \mathrm{~s}$ as illustrated in Fig. 7.

\subsection{Heating based on NLPQL algorithm}

This section presents the numerical simulation of heating operation based on the NLPQL optimization method. Using this method four parameters defining the time-optimum temperature were found: $T_{f_{1}}=63{ }^{\circ} \mathrm{C}$, $v_{T_{1}}=7.9 \mathrm{~K} / \mathrm{min}, T_{f_{2}}=440{ }^{\circ} \mathrm{C}, v_{T_{1}}=10.9 \mathrm{~K} / \mathrm{min}$. It was assumed that the pressure of working medium changes along the saturation line. The complete temperature and pressure history is shown in Fig. 8. Based on 


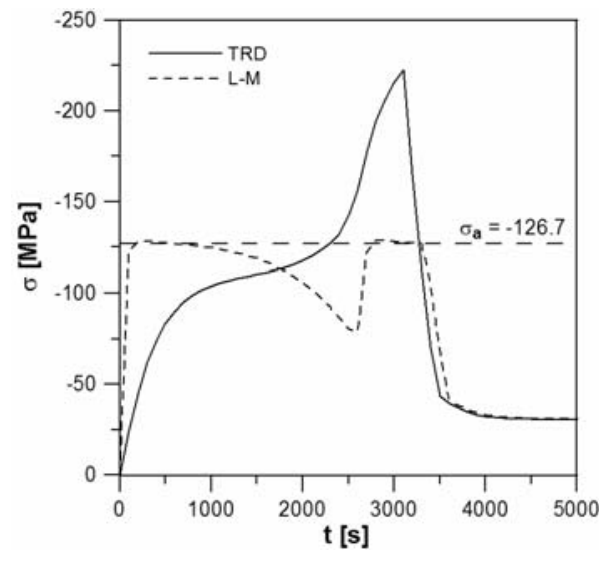

Figure 6. Comparison of maximum total compressive stress histories during the start-up process according to TRD 301 regulations and L-M method.
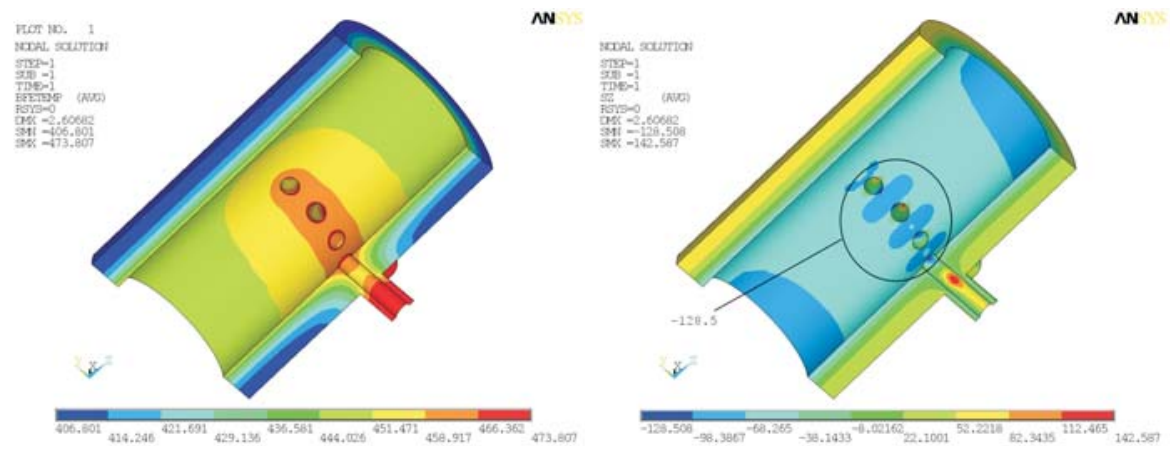

Figure 7. Temperature distribution in ${ }^{\circ} \mathrm{C}$ (left) and maximum total compressive stress, $\sigma_{z}$, distribution in $\mathrm{MPa}$ (right) on the inner surface at time $t=2800 \mathrm{~s}$.

the temperature and pressure histories the total time of heating process $t=3360 \mathrm{~s}$ was found. The maximum total stresses occurred on the inner surface at time $t=2900 \mathrm{~s}$ as illustrated in Fig. 9 .

During the heating process the total stresses in the whole construction element were monitored. Figure 10 presents the maximum total compressive stress history. 

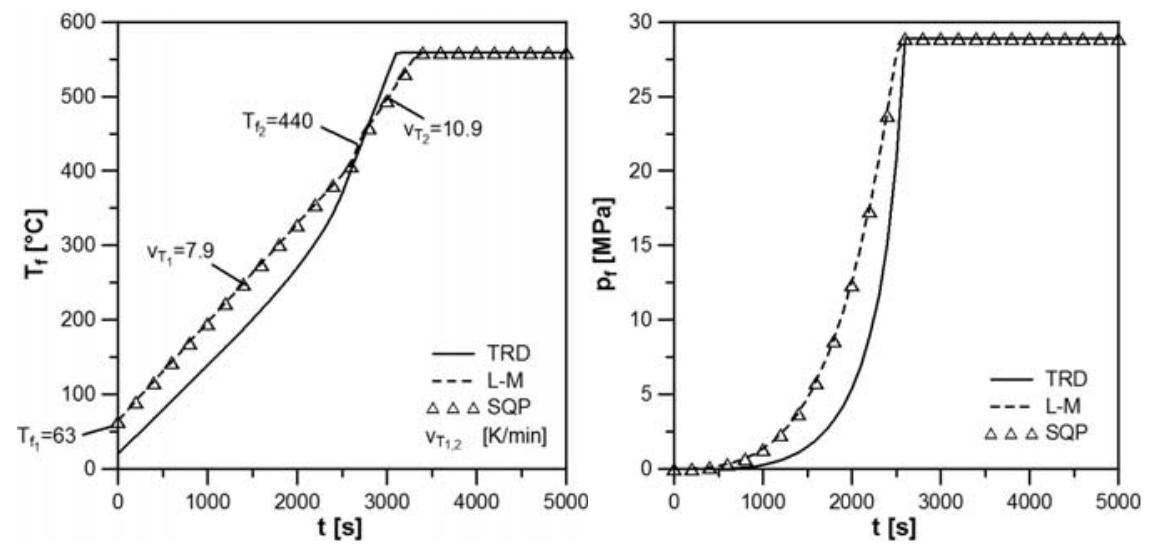

Figure 8. Comparison of temperature (left) and pressure (right) histories during the heating operation based on TRD 301 regulations, L-M algorithm and NLPQL algorithm.
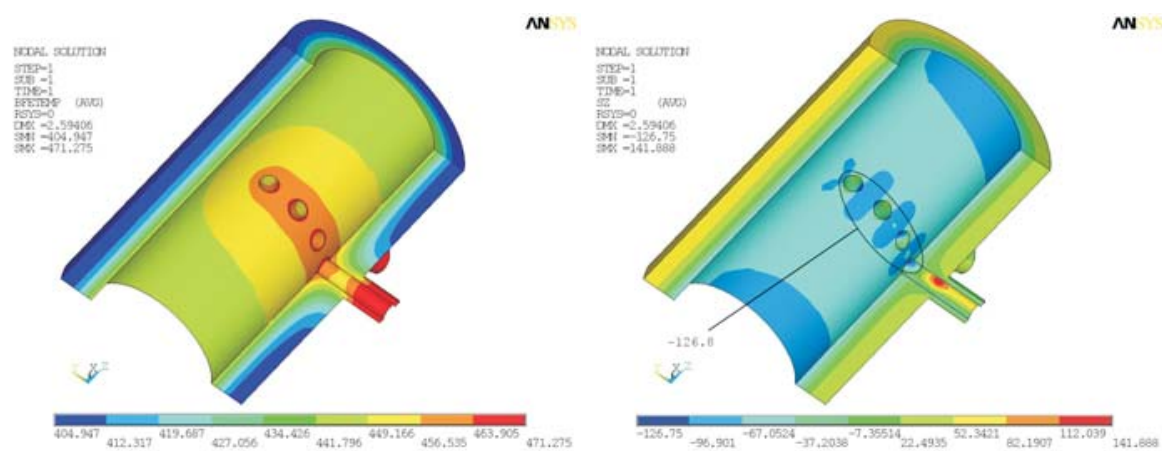

Figure 9. Temperature distribution in ${ }^{\circ} \mathrm{C}$ (left) and maximum total compressive stress, $\sigma_{z}$, distribution in $\mathrm{MPa}$ (right) on the inner surface at time $t=2900 \mathrm{~s}$.

\section{Conclusion}

Three methods, that allow us to find the optimum fluid temperature histories during heating operation of pressure components in supercritical steam power blocks were presented. The first method is based on the German boiler regulations TRD 301. In the second method, the LevenbergMarquardt algorithm was used. The third one is based on nonlinear programming by the quadratic Lagrangian algorithm. 


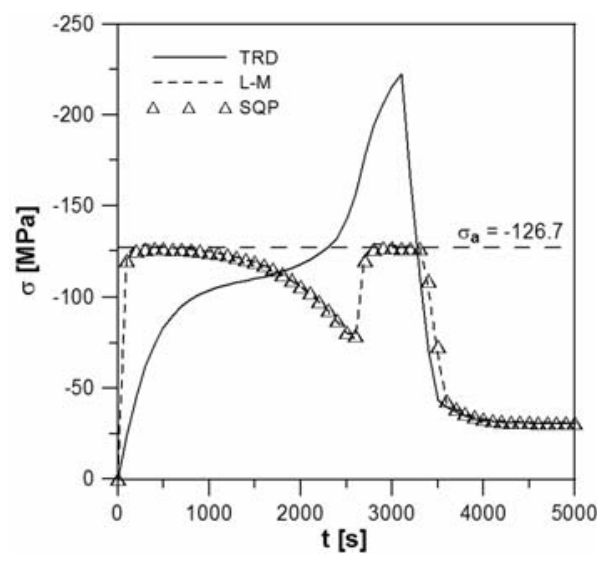

Figure 10. Comparison of maximum total compressive stress histories during the start-up process based on TRD 301 regulations, L-M algorithm and NLPQL algorithm.

All three methods were used to find optimum fluid temperature history during theof heating operation of the outlet header. The heating operation processed according to the German boiler regulations allowed to achieve nominal working parameters in the shortest time $3200 \mathrm{~s}$. However the maximum total compressive stresses exceeded the stress limit by more than $80 \%$. The start-up operation based on L-M and NLPQL algorithms took $3350 \mathrm{~s}$ and $3360 \mathrm{~s}$, respectively. In the case of L-M based optimization procedure the maximum total compressive stresses exceeded the allowable stress by less than $1.5 \%$. The NLPQL based heating procedure allowed to keep maximum total compressive stresses within allowable stress limit.

The presented optimization methods are of great practical significance and can be implemented in the industry, wherever the heating and cooling processes take place. They allow operating the steam power blocks in a cost-effective way and conform to the boiler regulations such as German boiler regulations - TRD 301.

Acknowledgements Part of this work was conducted within the confines of cooperation between TÜV NORD EnSys Hannover GmbH \& Co. KG and Cracow University of Technology. 


\section{References}

[1] TRD 301, Technische Regeln für Dampfkessel. Carl Heymans Verlag, Köln und Beuth-Verlag, Berlin 1986, 98-138.

[2] Duda P, Dwornicka R.: Optimization of heating and cooling operations of steam gate valve. Struct Multidisc Optim 40(2010), 529-535.

[3] Dzierwa P, Taler J.: A new method for determining allowable medium temperature during heating and cooling of thick walled boiler components. In: Proc. Congress on Thermal Stresses, 2. Taipei, Taiwan, 4-7 June, 2007, 437-440.

[4] Dzierwa P, Taler J.: A new method for optimum heating of steam boiler pressure components. Int. J. Energy Res. 34(2010), 20-35.

[5] Duda P, Rzasa D.: A new method for determining allowable medium temperature during transient operation of thick-walled elements in supercritical power plant. Arch. Thermodyn. 31(2010), 3, 55-72.

[6] Duda P, Rzasa D.: Numerical method for determining the allowable medium temperature during the heating operation of a thick-walled boiler element in a supercritical steam power plant. Int. J. Energy Res. 36(2012), 6, 703-709.

[7] Schittkowski K.: A FORTRAN subroutine solving constrained nonlinear programming problems. Ann. Oper. Res. 5(1986), 485-500.

[8] Press Wh, Teukolsky SA, Vetterling WT, Flannery BP.: Numerical Recipies in FORTRAN. The Art of Scientific Computing, 3 edn. Cambridge University Press: Cambridge 2007.

[9] Seber GAF, Wild CJ.: Nonlinear Regression. Wiley, New York 1989.

[10] Richter F.: Physikalische Eigenschaften von Stählen und ihre Temperaturabhängigkeit. Mannesmann Forschungberichte 930, Düsseldorf 1983.

[11] DudA P.: Solution of multidimensional inverse heat conduction problem. J. Heat Mass Transfer 40(2003), 115-122.

[12] Duda P, TAler J.: Solving direct and inverse heat conduction problems. SpringerVerlag, Berlin Heidelberg 2006.

[13] Levenberg, K.: A method for the solution of certain problems in least squares. Q. Appl. Math. 2(1944) 164-168. 